\title{
L'INTERPRETAZIONE KANTIANA DEL TESTO BIBLICO TRA FILOSOFIA DELLA STORIA E FILOSOFIA DELLA RELIGIONE
}

\author{
Fabrizio Lomonaco*
}

\begin{abstract}
RIASSUNTO
Negli scritti kantiani di filosofia della storia (1784-1786) la tradizione teologico-positiva ed il testo biblico, in particolare, rappresentano un punto di riferimento costante della ricerca critico-filosofica. Le pagine che seguono si propongono di vedere se il filosofo abbia, utilizzando il testo biblico, richiamato l'articolata trattazione filosofica del problema della storia e della conoscenza storica ai principi ed ai limiti del discorso critico fondamentale: ossia se si possa riconoscere la sacra Scrittura come criterio euristico adeguato a verificare le condizioni, i limiti e gli esiti della convergenza di criticismo e storicità, non sempre adeguatamente meditata dalla letteratura otto-novecentesca.
\end{abstract}

Parole-chiave: Kant. Filosofia della storia. Sacra Scrittura. Interpretazione teleologica.

\begin{abstract}
In the writings of Kant's philosophy of history (1784-1786) the positivetheological tradition and the biblical text, in particular, are a constant reference point in the search-critical philosophy. The following pages are intended to see if the philosopher has, using the biblical text, called the articulated discussion of the philosophical problem of the history and historical knowledge of the principles and limits of critical discourse fundamental: that if you can recognize the sacred Scripture as a heuristic criterion to verify the proper conditions, limitations and outcomes of the convergence of historical criticism, and not always properly thought out by the nineteenth-twentieth century literature.
\end{abstract}

Keywords: Kant. Philosophy of history. Sacred Scripture. Teleological interpretation.

\footnotetext{
* Professore Ordinario di Storia della filosofia nell'Università degli Studi di Napoli Federico II e Presidente del Consorzio interuniversitario "Civiltà del Mediterraneo". E-mail: fabrizio.lomonaco@unina.it
} 
Il mondo che-osserva Kant in un saggio del 1784 -all'osservazione empirica e immediata si presenta abbandonato al «caso sconfortante», «nell'insieme un miscuglio di stoltezza, di infantile vanità, spesso anche di infantile malvagità e mania di distruzione», non è solo un semplice, «informe aggregato di azioni umane», «determinate da leggi naturali universali così come ogni altro fatto della natura». Esso può essere, invece, identificato in un «sistema», non con «materiali (...) e metodi (...) ricercati (...) nella metafisica (...), nel gabinetto delle scienze naturali» ma attraverso l'idea della finalizzazione dell'ordine naturale e quella della totalizzazione del divenire in generale, essendo irragionevole «ammettere una finalità nella costituzione della natura e poi ammettere nell'insieme la mancanza di fini». Invero, nel concetto di uomo, essere finito e ragionevole, è inclusa la capacità di porsi dei fini e di agire conseguentemente, potendo egli «pensare» dal punto di vista etico-razionale anche ciò che gli risulta teoreticamente impossibile «conoscere» della e nella storia. Questa come scienza non è altro che meccanismo e causalità di avvenimenti, ma in quanto interpretazione è teleologia soggettiva e simbolica dei fatti. In fondo, la stessa «esperienza» del male, che rischia più di ogni altra di infirmare la legittimità e la possibilità di una «filosofia della storia», è ciò che dà senso al perenne umano differire dall'identità prerazionale, solo se inscritta in una rappresentazione teleologica della storia, in «un ordine per cui ciò che nei singoli individui si rivela confuso e irregolare, nella totalità della specie [può] riconoscersi come sviluppo continuato e costante, anche se lento, delle sue tendenze originarie» ${ }^{1}$.

\footnotetext{
${ }^{1}$ I. Kant, Idee zu einer allgemeine Geschichte in weltburgerlicher Absicht, in «Berlinische Monatsschrift», IV, (1784), pp. 385-411, poi in I. Kant's Gesammelte Schriften, herausgegeben von der Königlich Preussischen Akademie der Wissenschaften, Berlin 1910 sgg. (d'ora in avanti si cita con la sigla KGS), vol. VIII, pp. 18, 29, 25, 17, tr. it. di G. Solari in Id., Scritti politici e di filosofia della storia e del diritto, tradotti da G. Solari e G. Vidari, a cura di N. Bobbio, L. Firpo, V. Mathieu, Torino 1956, rist., ivi 1971 (d'ora innanzi si cita con Scritti politici), pp. 125, 124, 137, 133, 123 (d'ora in poi si cita con Idee, seguito dall'indicazione delle pagine dell'edizione tedesca e della traduzione italiana); Id., Recensionen von J. G. Herders Ideen zur Philosophie der Geschichte der Menscheit, in «Allgemeine Literaturzeitung», 1785, n. 4 e Beilage, I (pp. 17a-22b; n. 271, IV, pp. 15331560; d'ora in avanti si cita con Recensionen), poi in $K G S$, vol. VIII, p. 56, tr. it. in Scritti politici, p. 164.
} 
Ora, il fatto che l'interpretazione della storia risulti teleologica non è un esito casuale della riflessione di Kant. Né vi è alcuna controindicazione teorica o metodologica a considerare il senso del suo disegno di filosofia della storia in base ai concetti fondamentali della Kritik der praktischen Vernunft (1788) e nella Kritik der Urtheilskraft (1790), ma già esposti nella Kritik der reinen Vernunft (1781-1787), prima e durante l'elaborazione dei saggi di filosofia della storia degli anni Ottanta. In essi il dualismo tra noumeno e fenomeno, tra conoscere e pensare, posto a fondamento del discorso critico, resta insuperato ed insuperabile tanto da ridurre il concetto di «esperienza» a una modalità assunta solo per la spiegazione scientifica ${ }^{2}$. La mediazione che il finalismo elabora per riferire il complesso dei dati dell' «esperienza» a un ordine che in essa non può «apparire», è destinata a rivelarsi esterna ai termini da mediare e a sancire, perciò, «l'abisso senza fondo tra il casuale e il necessario», tra l'individuo e la specie morale, tra la «conoscenza storica» (cognitio ex datiis) e la «conoscenza razionale»

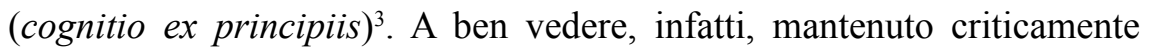
distinto dal mondo noumenico, pensabile solo dal punto di vista eticorazionale, il mondo dell' "esperienza» sensibile, conoscibile teoreticamente attraverso la connessione meccanico-causale dei fenomeni, indica soltanto la necessità teoretica dell'integrazione dell'osservazione causale con quella teleologica. Occorre servirsi, perciò, della nozione (soggettiva) di una causa intelligente del mondo («saggezza artistica»), per raggiungere la «massima unità sistematica dell'uso empirico della nostra ragione» e «considerare tutto ciò che può rientrare soltanto nel collegamento

2 Cfr. V. Delbos, Sur la noction de l'experience dans la philosophie de Kant, in Actès $\mathrm{du}$ Congrès International de Philosophie - Bibliothèque du Congrès International de Philosophie», Paris 1902, vol. IV, pp. 363-389; E. Cassirer, Das Erkenntnisproblem in der Philosophie und Wissenschaft der neueren Zeit, Berlin 19112, II, pp. 583-762, tr. it. di G. Colli, Torino 1971s, pp. 635-824. Sul tema e, più in generale, sulla letteratura di riferimento ho discusso nel mio contributo, Kant e il testo biblico. Le origini e gli esiti della filosofia "critica" della storia degli anni '80, in «Atti dell'Accademia di Scienze morali e politiche della Società Nazionale di Scienze, Lettere ed Arti in Napoli», XCIV (1983), pp. 267299. Fondamentale resta, naturalmente, l'aggiornamento (dal 1897) degli studi in «KantStudien». Philosophische Zeitschrift der Kant-Gesellschaft.

${ }_{3}^{3}$ I. Kant, Recensionen, p. 57, tr. it. cit., p. 165; Id., Kritik der reinen Vernunft (Riga 1787²), in KGS, vol. III, p. 540, tr. it. di G. Colli, Torino 1957, n. ed., Milano 1976, p. 809. 
dell'esperienza possibile, come se (...) l'insieme di tutte le apparenze (il mondo sensibile stesso) avesse al di fuori della sua estensione un unico fondamento, supremo e sufficiente a tutto, cioè una ragione, per così dire, autonoma, originaria e creativa, in riferimento alla quale noi indirizziamo ogni uso empirico (nella sua massima estensione) della nostra ragione, come se gli oggetti stessi fossero scaturiti da quell'archetipo di ogni ragione ${ }^{4}$. Ma questa prospettiva non fornisce alcun motivo per giustificare la finalizzazione di tutte le disposizioni naturali e del divenire in generale. Tale finalizzazione è, infatti, possibile solo sul presupposto che la natura in generale venga intesa come totalità teleologica, pensata, cioè, in base al concetto difine ultimo il quale non è un principio regolativo della valutazione di connessioni teleologiche entro la natura al fine della sua conoscenza teoretica, ma l'unico e ultimo criterio cercato fuori della natura, prescritto incondizionatamente per dare senso e valore all'uso pratico della ragione, al tipo di uomo e di mondo che essa tenta razionalmente di legittimare. Senza il riferimento all' «ideale del sommo bene, come fondamento determinante del fine ultimo della ragione pura», garanzia della coincidenza dei fini richiesti dalla legge morale con la connessione teleologica di tutte le cose, il mondo per l'uomo risulterebbe teoreticamente conoscibile nelle sue parti e, tuttavia, impensabile nella sua unità, incomprensibile in base ai princìpi della filosofia critica. Esposto inevitabilmente a tensioni profonde dai limiti della sua stessa ragione, l'uomo, per poter giudicare, dal punto di vista etico-razionale, sensata la sua attività e libera la sua esistenza deve aver fiducia in Dio, perché «senza un Dio e senza un mondo per noi adesso invisibile, ma sperato, le idee gloriose della moralità sono quindi certamente oggetti di applauso e di ammirazione, ma non già molle di propositi e di azioni, poiché non adempiono interamente al fine (...) determinato a priori dalla stessa ragione pura (...)». Questa ha bisogno di una certezza pratica che non nasce dal sapere o dal credere teoretici, ma da una «fede morale» ${ }^{5}$.

\footnotetext{
${ }^{4}$ Id., Kritik der reinen Vernunft, cit., p. 444, tr. it. cit., p. 681.

5 Ivi, pp. 522, 527, 531-538, tr. it. cit., pp. 785, 791, 797-805. Id., Eine Vorlesung Kants über Ethik, a cura di P. Menzer, Berlin 1924, poi in KGS, vol. XXVII, t. I, p. 306 e sgg. (t. II/2, pp. 1451, 1452 e sgg.), tr. it. di A. Guerra, Bari 1971, pp. 92, 93 e sgg.
} 
Una pura fede razionale è quindi la guida o la bussola con cui il pensatore speculativo può orientarsi durante le sue incursioni razionali nel territorio degli oggetti soprasensibili, e con cui l'uomo dotato di una ragione comune ma (moralmente) sana può tracciarsi, sia dal punto di vista teoretico che da quello pratico, una via pienamente adeguata allo scopo complessivo della sua destinazione; inoltre, questa fede razionale deve essere posta a fondamento di ogni altra fede, e di più, di ogni rivelazione ${ }^{6}$.

Solo sul presupposto di una tale fiducia vale, dunque, la tesi della finalità di tutte le disposizioni naturali e del divenire in generale da cui muove la filosofia della storia:

Se poi ci limitiamo a considerare per ogni dove da un lato la costituzione civile con le sue leggi, dall'altro i rapporti tra gli Stati (...) si scoprirà, come io credo, un filo conduttore, che (...) può giovare (...) anche $(\mathrm{e}$ ciò non si può fondatamente sperare senza il presupposto di un disegno della natura) (...) ad aprire una visione confortante dell'avvenire, nella quale la specie umana apparirà in lontananza come se avesse finalmente raggiunto quella condizione di vita in cui tutti i germi posti in essa dalla natura potranno conseguire un perfetto sviluppo e la sua destinazione qui sulla terra sarà pienamente adempiuta ${ }^{7}$.

Tuttavia, anche dagli esiti della riflessione sul significato e la legittimità filosofico-teleologica della storia è possibile riconoscere la compatibilità profonda tra criticismo e storicità, tra coscienza trascendentale e coscienza umana, entrambe interessate ed impegnate a salvaguardare il senso della razionalità dell'esistenza storica da ogni perfetta assolutizzazione che il criticismo kantiano non può celebrare, perché rispettoso, così in campo gnoseologico come in quello etico, dell'«individuo» concepito come valore supremo ${ }^{8}$.

${ }^{6}$ Id., Was heisst: Sich im Denken orientiren?, in «Berlinische Monatsschrift», VIII, (1786), pp. 304-330, poi in KGS, vol. VIII p. 142, tr. it. di G. De Flaviis, in Id., Scritti sul criticismo, Roma-Bari 1991, p. 24.

7 Id., Idee, p. 30, tr. it. cit., p. 138.

${ }^{8}$ Cfr. G. Solari, Il concetto di società in Kant (1934), poi in Id., La filosofia politica, cura di L. Firpo, Roma-Bari 1974, vol. II, p. 69. 
Oggetto della «fede morale» della ragione umana non è un essere che produce l'accordo tra condizionamento naturale e finalità morale ma un «ideale» che, nella sua perenne, noumenica inattingibilità regolativa, porta a coronamento l'intera conoscenza umana; un ideale che, però, garantisce solo la possibilità di pensare praticamente alla necessità di tale accordo, lasciando che a tale fine aspiri l'uomo stesso nell'agire secondo massime etico-razionali. Perciò la fiducia dell'uomo in Dio è, lontano da ogni forma di «sentimentalismo» fanatico e di «sofisticheria» teoretico-speculativa, l'adesione data direttamente dall'uso pratico della sua ragione a quegli «oggetti» e «problemi» che esaltano ed insieme tutelano nel mondo il senso della sua libera, responsabile condotta:

Il fine ultimo, cui tende la speculazione della ragione nel suo uso trascendentale, concerne tre oggetti: la libertà della volontà, l'immortalità dell'anima e l'esistenza di Dio. L'interesse semplicemente speculativo della ragione riguardo a questi tre oggetti è assai scarso (...). Anche questi però si rivolgono a loro volta ad un fine più lontano, ossia tendono a determinare che cosa si debba fare, se la volontà è libera, e se esistono un Dio ed un mondo futuro. Ora, dal momento che ciò riguarda il nostro comportamento rispetto al fine supremo, senza dubbio l'intenzione ultima della natura - che si prende cura saggiamente di noi - era propriamente rivolta, nel costituire la nostra ragione, soltanto all'interesse morale?

Ciò significa che il vero valore dell'esistenza umana non si identifica con il passivo godimento dei beni o con la ricerca dell'oziosa felicità ma si fonda sul senso autentico dell'azione, lontano da ogni pigrizia, da ogni «vano rimpianto» per una vita esente da cure e variata solo da giochi infantili in una fantasticante indolenza. Un rimedio ai dolori e alle pene prospettate dalla considerazione (teoretica) dei mali che travagliano il mondo va cercato dentro e non fuori la storia dell'uomo per il quale è un dovere aver fiducia nella storia ed essere soddisfatto della Provvidenza.

\footnotetext{
${ }_{9}^{9}$ I. Kant, Kritik der reinen Vernunft, cit., pp. 426, 518, 520, tr. it. cit., pp. 657, 780, 782; Id., Eine Vorlesung Kants über Ethik, cit., vol. I XXVII, t. I, p. 312 e sgg., 320-323 (t. II/2, p. 1456 e sgg., 1463-1465), tr. it. cit., pp. 100 e sgg., 111-114.
} 
Questa, impedendogli di «attribuire le proprie colpe a una colpa originaria dei suoi progenitori», lo invita a «contribuire per la sua parte e secondo le sue forze» al lento, ma progressivo miglioramento del genere umano ${ }^{10}$; miglioramento che è possibile rilevare interpretando il corso generale del mondo filosoficamente, non per smentire la «storia propriamente detta, concepita in maniera puramente empirica», bensì per ordinare teleologicamente i dati dell'«esperienza» storico-fenomenica. Tutto secondo il «filo conduttore a priori» nel disegno della «natura (o meglio della Provvidenza)», per scoprire se la stessa esperienza «riveli qualcosa di un siffatto andamento del disegno della natura (...), solo qualche piccolo dettaglio (...), anche i deboli indizi dell'avvicinamento [a questa età cosi felice] (...) per noi molto importanti». La scoperta del senso della storia, di tutti i suoi necessari, ineliminabili contrasti è manifestazione di un libero, cosciente e responsabile impegno dell'uomo, essere ragionevole e finito, interessato a orientarsi verso scopi futuri non assoluti o definitivi, assegnati in quanto razionalmente possibili e praticamente efficaci nella speranza «che, dopo qualche crisi rivoluzionaria di trasformazione, sorga finalmente quello che è il fine supremo della natura, cioè un generale ordinamento cosmopolitico, che sia la matrice, nella quale vengano a svilupparsi tutte le originarie disposizioni della specie umana $\rangle^{11}$. La riflessione filosofica pone, perciò, insieme alla questione del fine quella relativa all'identificazione globale, alla totalizzazione della storia. Tuttavia, se il fine postulato è solo praticamente sperato e non teoreticamente oggettivabile, cioè rappresentabile, il progetto di finalizzazione di tutte le disposizioni naturali e del divenire in generale, la cui «necessità» è colta nel «canone

10 Id., Muthmasslicher Anfang der Menschengeschichte, in «Berlinische Monatsschrift», VII, (1786), pp. 1-27, poi in KGS, vol. VIII, pp. 121, 123, tr. it. di G. Solari in I. Kant, Scritti politici, cit., pp. 208, 211 (d'ora innanzi si cita con Muthmasslicher, seguito dall'indicazione delle pagine dell'edizione tedesca e della traduzione italiana).

11 Id., Idee, pp. 27, 30, 28, tr. it. cit., pp. 134, 135, 138, 136. Cfr. I. P. Beade, Libertad y naturaleza en la Filosofia kantiana de la Historia, in «Daímon. Revista Internacional de Filosofía», n. 54 (2011), pp. 25-44 (con bibliografia aggiornata). Sull' «ideale cosmopolitico kantiano» cfr. dopo M. Mori (La pace e la ragione. Kant e le relazioni internazionali: diritto, politica, storia, Bologna 2008, spec. p. 231 e sgg.), le osservazioni di L. Tundo Ferente (Di una patria e del mondo. L'idea cosmopolitica fra utopia e realtà, Perugia, Morlacchi, 2011, pp. 63-809 sulla sua fortuna critica nella cultura contemporanea. 
della ragion pura» dalla religione proprio nel segno della speranza ${ }^{12}$, non totalizza la storia, non ne rappresenta la fine. A ben vedere, il problema dell'identificazione del fine del divenire storico con la sua stessa fine è un problema posto da Kant ma destinato a rimanere significativamente insoluto. Il senso della «storia generale dal punto di vista cosmopolitico» (allgemeine Geschichte in weltbürgerlicher Absicht) resta qualcosa di non necessario, di possibile nell'ordine dei fatti e di ideale nell'ordine dei progetti alla luce del significato critico di «una perfezione a cui l'uomo può sempre più avvicinarsi ma che non può mai raggiungere interamente» ${ }^{13}$. Risolvere tale tensione, ricondurre la «differenza» dall'irraggiungibile «ideale» in seno all'«identità» perfetta nel regno dell'assoluto definitivamente instauratosi sarà compito dell'idealismo postkantiano, a partire da Fichte.

Nella «filosofia» kantiana della storia, invece, l'aporetica ma ineludibile compresenza di due incompatibili tendenze, l'una affermazione dell'«esigenza» della totalizzazione dal punto di vista etico-razionale, l'altra negazione della stessa dal punto di vista teoretico resta il segno di un non risolvibile dualismo tra intelligibile e sensibile, tra necessità e libertà. Dato il quale, però, l' «esigenza» di totalizzazione diviene l'orizzonte in cui risulta possibile l'identificazione dell'effettiva storicità di ogni realtà della e nella storia. Questa non implica alcun atto o stato «perfetto», perché ha la propria autenticità nell'essere perennemente aperta a nuovi e mai definitivi progetti. Come in campo gnoseologico l'inconoscibile cosa in sé (il noumeno) non può essere eliminata, perché serve a impedire ogni assolutizzazione e a garantire significato al conoscere fenomenico, così solo all'interno dell'orizzonte della totalità, dell'«esigenza» critica dell'identificazione del differire visibile, la storia ha senso, risultando comprensibile quale perenne divergere dall' «ideale». Irriducibile a forme di totalizzazione assoluta e perfetta, è storia inconclusa dell'essere finito e ragionevole, collocato nell'universo del divenire e, insieme, appartenente

\footnotetext{
12 Id., Kritik der reinen Vernunft, cit., pp. 522-523 e sgg, tr. it. cit., pp. 785-786 e sgg.

13 Id., Anthropologie in pragmatischer Hinsicht, Königsberg 1798, in KGS, vol. VII, p. 200, tr. it. in Id., Scritti morali, a cura di P. Chiodi, Torino 1970, p. 621 (d'ora in poi si cita con Anthropologie, seguito dalla indicazione delle pagine dell'edizione tedesca e della traduzione italiana).
} 
al mondo dei valori etico-razionali in base ai quali egli deve tendere a identificare il divenire della storia, ad attribuirle criticamente senso nel «pensiero».

Ora, nel periodo immediatamente posteriore all'elaborazione dei saggi difilosofia della storia degli anni Ottanta, ad avvalorare l'interrogazione fondamentale sul destino finale del mondo, è l'interpretazione del Vangelo, l'«imperitura guida della vera saggezza, con la quale una ragione che perfezioni la propria speculazione non solo va d'accordo, ma dalla quale essa riceve anche una nuova luce riguardo a ciò che, sebbene abbia percorso interamente il suo campo, le rimane pur sempre oscuro e su cui abbisogna dunque di un ammaestramento ${ }^{14}$.

Nel saggio Über das Misslingen aller philosophischen Versilche in der Theodicee, pubblicato nel 1791, un anno dopo la Kritik der Urteheilskraft (1790) e poco prima di Die Religion innerhalb der Genzen der blossen Vernunft (1793) e dello scritto su Das Ende aller Dinge (1794), acquista un senso emblematico la valutazione che Kant dà della figura biblica di Giobbe. Più in generale si tratta dell'uomo che egli, messa definitivamente in discussione ogni filosofia dell'essere, pone al vertice della ricerca filosofica, presentando l'«antropologia» (che cos'è l'uomo?) quale luogo di lettura della possibile unione della «religione cristiana con la più pura ragione pratica» ${ }^{15}$. Già emerso nelle considerazioni filosofiche sulla storia biblico-congetturale delle origini, il problema della comprensione dei limiti dell'umana ragione, perennemente insoddisfatta nel suo uso speculativo e problematicamente interessata in quello pratico a risolvere l' «antinomia» tra fini morali e fini naturali ${ }^{16}$, è al centro delle osservazioni sulla presunta legittimità filosofica del discorso di teodicea che è la difesa «della saggezza suprema dell'autore dell'universo contro i quali

14 Lettera del $1^{\circ}$ marzo 1789 a H.J. Stilling, in Kant's Handschriftlicher Nachlass. Vorarbeiten una Nachträge, poi in $K G S$, vol. XXIII, p. 494, tr. it. in I. Kant, Epistolario filosofico. 1761-1800, a cura di O. Meo, Genova il melangolo, 1990, p. 174.

15 Lettera del 4 maggio 1793 a K.F. Staüdlin, in Kant's Briefwechsel (1789-1794), poi in $K G S$, vol. XI, p. 429, tr. it. in I. Kant, Epistolario filosofico. 1761-1800, cit., p. 320.

16 I. Kant, Kritik der praktischen Vernunft, Riga 1788, poi in KGS, vol. V, p. 113 e sgg., tr. it. in Id., Scritti morali, cit., p. 260 e sgg. 
quelle controfinalità vengono rivolte a titolo di obiezioni ${ }^{17}$. Nel ribadire le sue obiezioni teoretiche contro la teleologia e la teologia speculative - già esposte nell'Appendice alla «Dialettica trascendentale» della prima Kritik e successivamente nel saggio Über den Gebrauch teleologischer Principien in der Philosophie (1788), autentico preludio ai paragrafi della Kritik der Urtheilskraft (1790) sulla «Critica del giudizio teleologico»-, Kant denuncia le aporie della teodicea razionale, criticandone il tentativo di difendere la santità del Creatore in quanto legislatore, la sua bontà in quanto reggitore dell'universo e la sua giustizia in quanto giudice. Avversa l'illegittimo argomentare rispettivamente contro la realtà del male morale (il peccato), del male fisico (il dolore) e del male sotto forma di impurità, «come non corrispondenza tra i crimini e le pene». In ognuno di questi tre casi il tentativo della teodicea speculativa è destinato a fallire, perché vano risulta lo sforzo di rivolgersi al mondo quale oggetto d'esperienza per trarre da esso un significato morale:

Quale altro filo conduttore è dato infatti alla ragione per le sue ipotesi teoretiche, se non la legge di natura? (...); la nostra ragione è del tutto incapace di cogliere il rapporto esistente tra un mondo quale è quello che possiamo conoscere sempre e solo attraverso l'esperienza e la saggezza suprema (...). Per por fine una volta per sempre a questo processo, occorre quindi dimostrare ancora che siamo in grado di raggiungere perlomeno una saggezza negativa, prendendo atto della necessità di porre un limite alle nostre pretese riguardo a ciò che non è alla nostra portata; e questo lo si può fare benissimo.

${ }^{17}$ Id., Über das Misslingen aller philosphischen Versuche in der Theodicee, in «Berlinische Monatsschrift », XIII (1791), pp. 194-225, poi in KGS, vol. VIII, p. 255, tr. it. di di G. De Flaviis, in Id., Scritti sul criticismo, cit., p. 133 (d'ora innanzi si cita con Über das Misslingen, seguito dall'indicazione delle pagine dell'edizione tedesca della traduzione italiana). Con riferimento ai temi e agli scritti di filosofia della storia si vedano R. Langthaler, Kant "Weltbegriff der Philosophie" und die "Rangordnung der menschlichen Zwecke", in Studi italo-tedeschi. XXV Simposio internazionale sul tema "Immanuel Kant (17241804) nel $200^{\circ}$ annibersario della morte" (Merano 2004), Trento 2005, pp. 317-337 e C. La Rocca, Illuminismo e forme di razionalità. Kant e la ragione come fine, in Il realismo della ragione. Kant dai lumi alla filosofia contemporanea, a cura di S. Poggi, Milano-Udine 2012, pp. 263-284. 
La filosofia «critica» non smentisce una finalità nella e della natura ma nega che da questa finalità naturale si possa risalire a quella morale e alla teologica che non presentano alcuna «speranza» per la ragione teoretica, né permettono un allargamento dell'area conoscitiva, ravvisando solo nella postulazione pratica un suo legittimo modo di essere razionale:

Noi possediamo infatti un concetto di saggezza artistica qual essa si manifesta nell'organizzazione di questo mondo, un concetto dotato di una realtà oggettiva ch'è sufficiente alla nostra facoltà razionale speculativa per giungere ad una fisico-teologia. Possediamo, altresì, nell'idea morale della nostra propria ragion pratica, il concetto di una saggezza morale che potrebbe essere stata posta in un mondo in generale da un autore perfettissimo. Quel che però no abbiamo né possiamo sperare di riuscir mai ad avere è un concetto della concorde unità di questa saggezza artistica con la saggezza morale in un mondo sensibile ${ }^{18}$.

Nell'uomo, esposto drammaticamente ai rischi dell'«illusione trascendentale» che «non può assolutamente essere evitata» ${ }^{19}$, l'eterogeneità esistente tra «conoscere» e «pensare», tra coscienza naturale e coscienza etico-religiosa, riproduce l'«antagonismo originario e insanabile» tra intelligibile e sensibile, tra mondo morale e universo naturale. L'insuccesso de jure di ogni teodicea razionale rende, così, insufficiente la difesa contro l'azione malvagia, difesa possibile contro tale contraddittorio vizio intrinseco solo se è interiore e non «esteriore all'azione, esteriore al contenuto di essa $»^{20}$. Non a caso, a sottolineare l'infondatezza di ogni apertura teoretica nel mondo etico-teologico è la stessa scelta ermeneutica di Kant per un'interpretazione analogica della sacra Scrittura che, priva di ogni metafora «organicistico-tipologica», centralissima, invece, nell'ermeneutica di Hamann e di Herder, impegna il filosofo di

\footnotetext{
18 I. Kant, Über das Misslingen, pp. 256, 262, 263, tr. it. cit., pp. 138, 139-140.

19 Id., Kritik der reinen Vernunft, cit., pp. 234, 236, tr. it. cit., pp. 359, 362.

20 Così G. Capograssi, Analisi dell'esperienza comune, Roma 1930, p. 158, poi in Id., Opere, Milano 1959, vol. II, p. 164.
} 
Königsberg a riflettere non tanto sul testo sacro come documento storico ${ }^{21}$, quanto sul senso filosofico fondamentale dell'uso del linguaggio biblico come criterio interpretativo per verificare «se il cammino che la filosofia percorre mediante i concetti s'accorda con quello della storia $\rangle^{22}$. Eppure, tale proposta "analogica" resta radicalmente distinta da quella fatta valere dalla fisico-teologia per garantire, mediante la nozione (soggettiva) di una «saggezza artistica», di una causa intelligente del mondo, unità e senso teoretici all'indagine del Naturforscher. L'interpretazione critico-filosofica del «documento» biblico si radica in un'ermeneutica di tipo simbolico, per «pensare» l'inconoscibile. Testimonianza dei limiti dell'uomo, della sua condizione d'essere ragionevole e finito, il linguaggio simbolico deve utilizzare quello teoretico, al fine di «esibire» indirettamente ciò che può essere pensato nella forma dell'analogia. Questa, assunta in un'accezione derivata dal linguaggio matematico, non mira all'individuazione ontologica dei termini che entrano in rapporto (analogia entis, ma al riconoscimento del senso del rapporto stesso (analogia attributionis), essendo solo la «somiglianza perfetta di due rapporti tra cose del tutto dissimili». Nel congedo definitivo da ogni ontologia, la relazione analogico-simbolica è, allora, un particolare tipo di «conoscenza», interessata a giustificare una

\footnotetext{
${ }^{21}$ J. G. Herder, Ideen zu einer Philosophie der Geschichte der Menschheit, Riga Leipzig 1784-1791, in Sämmtliche Werke, a cura di B. Suphan, Berlin 1877-1913, poi rist. fot., Hildesheim 1967, 33 vol., vol. XIII spec. pp. 396-439, tr. it. di V. Verra, Bologna 1971, parte II, libro X, pp. 249-257. Del Verra si vedano i fondamentali contributi ora raccolti in volume Linguaggio, mito e storia. Studi sul pensiero di Herder, a cura di C. Cesa, Pisa 2006. Per una più ampia informazione sul tema sia consentito rinviare ai miei contributi Verità religiosa e verità storica nella filosofia del linguaggio di Herder fino al 1783 prima della Ideen, in «Atti dell'Accademia di Scienze Morali e Politiche della Società Nazionale di Scienze, Lettere e Arti in Napoli», vol. XCIII (1982), pp. 157-199; La polemica KantHerder sull'origine e il destino della storia umana, in «Prospettive Settanta», n. s., XIII (1991) 1, pp. 76-102 e La question de l'origine du langage dans l'Abhandlung de Johann Gottfried Herder (traduit par E. Martinelli), in Interdit. Essays on the origin of Language (s), a cura di M. Castagna, in «Sistemi Linguistici», 1 (2012), pp. 51-64 (con relativa bibliografia). Si vedano anche gli aggiornati studi di R. Thurnher, Kant und Herder über den Sinn der Geschichte, in Studi italo-tedeschi, cit., pp. 239-253 e P. Rehm, Le conflit entre Kant et Herder sur la philosophie de l'histoire, in Kant et les lumières européennes. Actes du VII Congrès de la Société d'Études Kantiennes de Langue française organisé à Naples (20-22 octobre 2005), sous la direction de L. Bianchi. J. Ferrari et A. Postigliola, Paris-Naples 2009, pp. 141-147.
}

22 I. Kant, Muthmasslicher, p. 110, tr. it. cit., p. 196. 
determinata immagine del mondo e dell'uomo che la istituisce in funzione delle sue esigenze etico-razionali, nella consapevolezza che non è lecito «spacciare i confini della nostra ragione per confini della possibilità delle cose stesse $\rangle^{23}$. Del resto, è proprio la ricostruzione analogico-simbolica della storia biblica delle origini a insegnare che il male nel mondo è compreso non a partire da Dio ma dall'uomo, il cui vero discorso di teodicea deve articolarsi nel dominio non «d'una ragione raziocinante (speculativa), bensì di quella offerta da una ragione pratica dotata d'un'autorità». A differenza della teodicea dottrinale, un «libro chiuso» che non saprà mai «l'intenzione ultima di Dio», quella autentica, che riconosce all'idea del sommo bene la santità, la giustizia e la bontà, deve esistere come «esigenza» del vivere morale. Essa, proprio per garantire la speranza nel regno dei fini che la moralità esige e difenderne la realizzazione nell'idea teologica, va mantenuta nella sua santità, non discussa teoreticamente, ma sofferta ed adorata ${ }^{24}$.

Nella parte seconda del saggio la polemica nei confronti dell'ispirazione non propriamente etica di ogni teodicea speculativa trasferita dal piano teoretico a quello etico-religioso - si incarna nell'esame della figura biblica di Giobbe. In Kant la sua tragica esistenza, segnata dalla drammatica contraddizione del giusto che non è felice, conseguente alla radicale antinomia della ragione umana nel suo uso pratico, non si risolve nel sistema «filosofico» di un'armonia leibnizianamente prestabilita. Al centro del suo discorso di teodicea non c'è Dio ma la volontà dell'uomo Giobbe di discutere con Dio e di Dio. Diversamente dai suoi «amici» che per decifrare, conoscere e spiegare l'impenetrabile potenza divina tentano impunemente di ragionare e di far uso della «dottrina», Giobbe vive la teodicea autentica. Egli non fonda la sua moralità su di una

${ }^{23}$ Id., Prolegomena zu einer jeden künftigen Metaphysik, die als Wissenschaft wird auftreten können, Riga 1783, poi in $K G S$, vol. IV, pp. 357 e nota, 351, tr. it. di P. Carabellese, rivista da R. Assunto, intr. e revisione di H Hohenegger, ed. V, Roma-Bari 2009, pp. 239, 225. Dell'uso della conoscenza analogico-simbolica nell'interpretazione della sacra Scrittura Kant tratta nella Die Religion innerhalb der Grenzen der blossen Vernunft, Königsberg 1793 (poi in $K G S$, vol. VI, p. 64 nota, tr. it. di P. Chiodi in I. Kant, Scritti morali, cit., pp. 385-387 nota; d'ora in poi si cita con Die Religion e l'indicazione delle pagine dell'edizione tedesca e della traduzione italiana) e nella Anthropologie (p. 192, tr. it. cit., p. 613).

24 I. Kant, Über das Misslingen, p. 264, tr. it. cit, pp. 141, 142. 
fede teoreticamente intesa, ma, arresosi alla saggezza ed alla maestà impenetrabile di Dio, si dichiara per il «sistema dell'incondizionatezza del decreto divino. "Egli è unico — afferma — ed egli fa come vuole». Nell' eroe biblico la fede, nata «da una sì profonda risoluzione dei suoi dubbi, ossia semplicemente convincendosi della sua ignoranza», è la «prova di fondare non la sua moralità sulla fede, bensì la fede sulla moralità»: quella fede che «per quanto debole possa essere, è però d'un genere più puro e più autentico; un genere di fede che fonda una religione della buona condotta, e non già interessata prioritariamente al favore divino $»^{25}$. Incomprensibile secondo i princìpi di un'interpretazione soltanto erudito-filologico-storica, inesplicabile secondo i valori dell' «umanesimo ebraico» di Hamann e di Herder $^{26}$, il libro di Giobbe non è uno dei tanti «documenti» della storia umana delle origini, ma la testimonianza di una fondamentale esperienza religiosa, l'unica capace, nella sua struttura morale, di conferirle senso ed unità. A chi, presumendo di «conoscere» la fine di tutte le cose e di sentirsi giustificato da ciò a non ascoltare la voce della coscienza morale (das Gewissen), acquista un pericoloso abito alla «falsità e alla slealtà, principale difetto della natura umana», si contrappone la scelta di Giobbe. Essa è quella della coscienza leale e sincera di ogni uomo retto, interessato a seguire, contro gli astratti e falsi articoli di fede, «la semplice sincerità del cuore e non il privilegio dell' intellezione, l'onestà che ci porta a confessare apertamente i nostri dubbi, la ripugnanza a simulare convinzioni da noi non sentite $(\ldots) \gg\rangle^{27}$. Eppure, il privilegiamento di questa connessione fondamentale di fede, lealtà e sincerità, cui è affidata l'interpretazione del racconto e della figura di Giobbe, resta nella prospettiva dell'impossibilità teorizzata, di qualsiasi totalizzazione assoluta, definitiva della storia. Il movimento dell'umanità verso la piena realizzazione del «regno di Dio sulla terra» è movimento perennemente inconcluso ed interrotto, fondato

\footnotetext{
${ }^{25}$ Ivi, pp. 265, 267, tr. it. cit., pp. 142, 143-144.

26 H.J. Kraus, Geschichte der historisch-kritischen Erforschung des Alten Testaments von der Reformation bis zur Gegenwart, Neukirchen 1956, tr. it. di G. Martinetto, Bologna 1975, pp. 183-210.

27 I. Kant, Das Ende aller Dinge, in KGS, vol. VIII, pp. 325-339, tr. it. di G. De Flaviis, in Id., Scritti sul criticismo, cit., pp. 239-254; Id., Über das Misslingen, pp. 266-267, tr. it. cit., pp. 144, 143.
} 
sulla coscienza dell' «impenetrabilità» del «male radicale della natura umana», sull'irriducibilità della «lotta fra il principio buono e il cattivo per il dominio sull'uomo» ${ }^{28}$, sull'impossibilità che il termine ultimo di approdo, il bene, si costituisca immediatamente senza fratture. Queste risultano, infatti, ineliminabili nell'aspirazione a una «salvezza» che, già imperiosamente annunziata ma non ancora compiutamente realizzata, è destinata a contrassegnare il particolare equilibrio della filosofia kantiana della religione, il suo complesso tessuto critico-problematico, mosso tra il riconoscimento della purezza e dell'universalità della fede razionale, inscritta nel cuore dell'uomo, e l'affermazione dell'inevitabile fede storica: quella contenuta nei libri sacri, in grado di rivelare, «schematizzandola», la religione razionale nella sua interezza, in grado di fornire alla ragione quelle «dottrine» che essa non può elaborare da sé, ma di cui ha un assoluto bisogno per il suo corretto esercizio pratico ${ }^{29}$.

Se scopo del discorso sulla «religione nei limiti della semplice ragione» è quello di vedere il massimo di religione che la ragione, nel suo uso pratico, concede come speranza, per la vita morale autentica, non c'è, allora, che un modo, perché l'uomo possa, nei limiti del suo linguaggio razionale e simbolico, aprirsi al vero significato del «documento» biblico. Esso consiste nel conferire alle «regole pratiche universali di una religione razionale pura (die reine Vernunftreligion) (...) il principio ultimo di ogni

${ }^{28}$ Id., Die Religion, pp. 19 e sgg., 57 e sgg., 74, 183-184, 190 n e sgg., tr. it. cit., pp. 337 e sgg., 377 e sgg., 397, 398, 514, 521 nota e sgg. Cfr. W. Henckmann, Das “krumme Holz" und die Aufgabe der Moralisierung, in Studi italo-tedeschi, cit., pp. 207-222.

29 Già in una lettera del 28 aprile 1775 a J.C. Lavater (Kant's Briefwechsel (1747-1788), poi in $K G S$, vol. X, pp. 175-179, tr. it. in I. Kant, Epistolario filosofico. 1761-1800, cit), il filosoofo della Kritik, paragonando la propria condotta a quella dell'eroe biblico Giobbe, simbolo della «buona condotta di vita e della purezza delle intenzioni nella fede», fissa lucidamente i principi della legittima ermeneutica biblica, distinguendo tra l'insegnamento fondamentale (die Grundlehre) e quello ausiliario (die Hulfslehre) del Vangelo: il primo si fonda sulla «fede morale», sulla «fidu $\neg$ cia incondizionata nell'aiuto divino riguardo ad ogni bene che, ad onta dei nostri sforzi più coscienziosi, non è in nostro potere»; il secondo sulla «venerazione di questo maestro (...) adulandolo ed innalzandogli lodi (...). Tuttavia questo metodo era meglio adatto a quei tempi (...) che ai nostri, in cui era necessario che agli antichi miracoli se ne contrapponessero di nuovi, che ai precetti ebraici si contrapponessero quelli cristiani» (ivi, pp. 89, 90-91, 92). Sul tema si vedano le aggiornate e interessanti osservazioni di P. Koenig, Il Giobbe dell'Illuminismo. Kant e il problema della teodicea, in Kant et les lumières européennes, cit., pp. 227-235. 
interpretazione della Scrittura», al fine di stabilire quanto «nel testo della religione che è creduta rivelata, la Bibbia, può essere riconosciuto anche tramite la sola ragione ${ }^{30}$. In sede filosofico-razionale la dimensione storica del testo biblico per avere senso deve paradossalmente annullarsi. La fede che incrementa nell'uomo la moralità non può avere alcuna fondazione storica, essendo la «legislazione statutaria (che suppone una rivelazione) (...) casuale (zufallig), quindi priva della garanzia che sia giunta o giunga a ogni uomo, perciò non può essere ritenuta obbligatoria per ogni uomo in generale», «non è partecipabile universalmente in modo convincente». La componente pratico-morale dell'ermeneutica razionale, affidata, contro le pretese del «teologo biblico», alle cure del filosofo interprete della religione, è tanto decisiva da ricondurre «tutto ciò che può essere contenuto nella Scrittura per la fede storica alle regole e ai motivi della fede morale». In essa non suscita più interesse l' «elemento teoretico della fede ecclesiastica» né il sentimento (das Gefühl), introdotto esteriormente in modo puramente gratuito, né, infine, la dimensione erudito-filologica del testo sacro, preoccupata di difendere dogmaticamente l'articolo di fede senza incrementare motivi utili al miglioramento morale dell'uomo, la cui religione appartiene all'area del «fare» ${ }^{31}$. Perciò, interpretabili e comprensibili nel «documento» biblico sono soltanto quelle «esperienze» che hanno per l'uomo un senso non letterale, non storico; e ciò non perché le parole siano insignificanti o vane, ma perché la pienezza del loro significato le trascende continuamente e soltanto in tale trascendere risultano praticamente possibili ed efficaci. Tuttavia, la possibilità dello iato e del contrasto tra l'elemento storico-rivelato e quello etico-razionale pone, in linea di principio, anche la questione della coincidenza possibile fra i due elementi, rendendo, così, problematici i confini all'interno dei quali dovrebbe rimanere la considerazione "religiosa" della ragione:

30 I. Kant, Die Religion, pp. 3 e sgg., 110, 112, tr. it. cit., pp. 323 e sgg., 435, 438; Id., Der Streit der Facultäten, Königsberg, 1798, poi in KGS, vol. VII, p. 6 nota, tr. it. di D. Venturelli, Brescia 1994, p. 58 nota.

${ }^{31}$ Id., Die Religion, pp. 104, 109, 110, 111, 112, 113, 114, tr. it. cit, pp. 430, 435, 436, 437, 438, 439, 440, 441; Id., Der Streit der Facultäten, cit., parte I: «Der Streit der philophischen Facultat mit der theologischen», pp. 41 e sgg., 61 e sgg., tr. it cit., pp. 103 e sgg., 130 e sgg. 
Da questo punto di vista si potrà intraprendere un secondo tentativo, cioè partire dalla rivelazione ammessa come tale (...) per vedere se, per questa via, si è ricondotti al medesimo sistema razionale puro della religione (...). Se il tentativo dovesse riuscire, si potrà sostenere che fra la ragione e la Scrittura esiste non solo compatibilità, ma anche unione, di modo che chi segue l'una (sotto la guida dei concetti morali) non potrà evitare di accordarsi anche con l'altra. Ma nel caso che il tentativo fallisse (...) una religione e un culto (...) dovrebbero essere agitati e mescolati sovente perché sia possibile vederli uniti per breve tempo; ma si separerebbero ben presto di nuovo, come l'olio e l'acqua lasciando galleggiare al di sopra l'elemento morale puro (cioè la religione razionale $)^{32}$.

Il problema non è, quindi, semplicemente quello di rifiutare, attraverso la difesa delle certezze della fede razionale, l'interpretazione storico-letterale della Scrittura. Occorre, infatti, verificare se sia possibile una Vernunftreligion anche nell' «esegesi» del dato storico che, in quanto «ipotiposi», «veicolo» dell'autentica religione morale, rappresenti una simbolizzazione propedeutica, necessaria al destarsi stesso della coscienza etico-religiosa:

Se è ormai chiaro che una fede ecclesiastica statutaria non è un'aggiunta indispensabile alla fede religiosa pura, quale veicolo e mezzo d'unione pubblica degli uomini a favore di questa fede, bisogna anche riconoscere che l'immutabile conservazione di questa fede ecclesiastica, la sua diffusione universale uniforme, nonché il rispetto per la rivelazione in essa ammessa, difficilmente possono essere assicurate mediante la tradizione, ma richiedono la Scrittura che, a sua volta, in quanto rivelazione, dev'essere oggetto di profonda venerazione da parte dei contemporanei e delle successive generazioni come ciò di cui gli uomini hanno bisogno per essere certi dei loro doveri di culto.

${ }^{32}$ Id., Die Religion, pp. 12, 13, tr. it. cit., «Prefazione alla seconda edizione», pp. 333, 334. 
Compito del filosofo-interprete della religione non è, allora, la distruzione, l'annullamento del «positivo» della religione, bensì la realizzazione di una sua lettura razionale mediante una scienza della Scrittura, necessaria non solo per dimostrarne l'autenticità ma per la sua stessa interpretazione:

È dunque indispensabile che l'interprete ne conosca la lingua in modo approfondito e sia inoltre in possesso di un'estesa conoscenza storica e critica, al fine di scoprire nelle situazioni nei costumi e nelle opinioni dell'epoca (nella fede popolare) gli strumenti per favorirne la comprensione da parte della comunità ecclesiastica ${ }^{33}$.

Eppure, a ben vedere, se nel «sapere» storico non si rivela semplicemente il fallimento di ogni mediazione tra «visibile» ed «invisibile», tra storia e ragione, l'impossibilità stessa di una considerazione «filosofica» della religione, la commisurazione teorizzata tra la costitutiva storicità della testimonianza biblica ed i princìpi della fede religiosa pura è destinata a restare non assoluta, profondamente problematica; è radicata, cioè, nel non risolto e non risolvibile dualismo critico tra sensibile e intelligibile, ripetuto, a suo modo, dall'insuperabile opposizione tra la Vernunftreligion e la Offenbarung contro ogni forma astratta ed assoluta di totalizzazione del divenire. La «vittoria del principio buono sul cattivo e la fondazione di un regno di Dio sulla terra», la realizzazione «pubblica» di una «società etico-civile o (di) una comunità etica» che, decretando la scomparsa della forma stessa di «Chiesa», dovrebbero portare alla «fine del mondo», all'annullamento del suo divenire «visibile», non possono risultare conclusivi ${ }^{34}$. Perennemente ostacolati e rimessi storicamente in discussione, essi restano un «ideale» che, mai pienamente realizzabile, serve a porre la «questione della differenza fra la fede razionale e quella storica», giammai a superare tale differenza, mantenuta e riproposta, non a caso, nel capitolo IV di Die Religion ( «Intorno al culto vero e al culto falso sotto il dominio del principio buono») $)^{35}$.

\footnotetext{
${ }^{33}$ Ivi, pp. 106-107, 112-113 tr. it. cit., pp. 432, 439.

34 Ivi, pp. 93, 94, 134, tr. it. cit., pp. 417, 418, 463.

${ }^{35}$ Ivi, pp. 124, 151 e sgg., tr. it. cit., pp. 452, 479 e sgg.
} 
Allora, l'accusa frequentemente rivolta a Kant di mancanza di senso storico, di disinteresse per la storia non sembra cogliere nel segno, perché ignora la sostanziale complessità, dal punto di vista critico della riflessione sulla storia umana. Interpretando il testo biblico, filosofia della storia e filosofia della religione pongono, nei loro rispettivi ambiti, le premesse per la fondazione di un nuovo tipo di «universalismo». Eppure, in base ai presupposti del discorso critico, non lo fondano, non possono fondarlo, preoccupate come sono di salvaguardare la loro razionalità, la coscienza delle loro problematiche e non totalizzabili relazioni con l'universo fenomenico. Garantito in campo gnoseologico dal non eliminato noumenon, dall'inconoscibilità della cosa in sé e, in quello etico, dalla perenne inattingibilità dell'«ideale» regolativo, l'universalismo proposto da Kant è «universalizzazione», è «universalizzabilità». Questa, contro ogni perfetta assolutizzazione senza residui delle energie e delle ragioni individuali, mira a riportare «la normatività all'azione individuale», a salvare «le ragioni dell'essere normativo nell'esistenza delle azioni». Si stabilisce, così, «un rapporto nuovo tra le stesse individualità, in una coesistenza destinata a oltrepassare gli schemi del "regno dei fini" per aprirsi all'attiva razionalità di una convivenza dinamica meno armonizzata, ma più attenta a intendere il valore delle formazioni storiche molteplici in cui le forme dell'eticità possono realizzarsi» ${ }^{36}$.

Data de registro:21/05/2013

Data de aceite: $08 / 07 / 2013$

36 P. Piovani, Giusnaturalismo ed etica moderna, Bari 1961, p. 148 (poi nuova ed., a cura di F. Tessitore, con due note di N. Bobbio e G. Calogero, Napoli 2000); Id., Oggettivazione etica e assenzialismo, a cura di F. Tessitore, Napoli 1981, p. 109 (poi in Per una filosofia della morale, a cura di F. Tessitore, Milano 2010). 